\title{
A Cysteine-Rich Receptor-Like Protein Kinase CaCKR5 Contributes to Immune Defense Against Ralstonia Solanacearum in Pepper
}

\section{Shaoliang Mou}

Fujian Agricultural University: Fujian Agriculture and Forestry University

\section{Feng Gao}

Fujian Agriculture and Forestry University

Tingting Zhang

Fujian Agriculture and Forestry University

\section{Weihong He}

Fujian Agriculture and Forestry University

\section{Qianqian Meng}

Fujian Agriculture and Forestry University

\section{Deyi Guan}

Fujian Agriculture and Forestry University

Shuilin He ( $\nabla$ shlhe201304@aliyun.com )

Fujian Agriculture and Forestry University

\section{Research article}

Keywords: Capsicum annuum, CaCRK5, Ralstonia solanacearum, Resistance

Posted Date: December 1st, 2020

DOl: https://doi.org/10.21203/rs.3.rs-113516/v1

License: (c) (i) This work is licensed under a Creative Commons Attribution 4.0 International License. Read Full License

Version of Record: A version of this preprint was published at BMC Plant Biology on August 19th, 2021. See the published version at https://doi.org/10.1186/s12870-021-03150-y. 


\section{Abstract}

Background: Cysteine-rich receptor-like kinases (CRKs) represent a large subfamily of receptor-like kinases and have important roles in numerous different physiological processes in plants.

Results: CaCRK5 transcripts were observed to accumulate after the inoculation of $R$. solanacearum and treatment with salicylic acid. The fusion between CaCRK5 and the green fluorescence protein was targeted to the plasma membrane. Suppression of CaCRK5 via virus-induced gene silencing made pepper plants significantly susceptible to $R$. solanacearum infection, which was accompanied by decreased expression of defense related genes CaPR1, CaSAR8.2, CaDEF1 and CaACO1. Overexpression of CaCRK5 in tobacco conferred increased resistance against $R$. solanacearum. Furthermore, electrophoretic mobility shift assay and chromatin immunoprecipitation with quantitative real-time PCR analysis verified that a homeodomain zipper I protein CaHDZ27 can bind to the CaCRK5 promoter, and directly active its expression. Yeast two-hybrid and bimolecular fluorescence complementation analyses suggested that CaCRK5 could heterodimerize with the homologous member CaCRK6 in the plasma membrane.

Conclusions: Our data indicated that CaCRK5 played a positive role in pepper resistance against $R$. solanacearum infection.

\section{Background}

When plants are attacked by pathogen, plant immune receptors can detect pathogen infection and elicit a battery of defense responses. As the first line of plant defense, pattern recognition receptors (PRRs) can perceive pathogen-associated molecular patterns (PAMPs) that are released during pathogen infection and activate plant immunity [1]. More researches uncovered that most surface-localized PRRs identified so far are receptor-like kinases (RLKs) in plants [2, 3]. Well-characterized PRRs include the Arabidopsis leucine-rich repeat (LRR) receptor kinases FLAGELLIN SENSING 2 (FLS2) or EF-TU RECEPTOR (EFR), which can recognize bacterial flagellin [4] or EF-Tu [5], respectively. RLKs comprise a superfamily of transmembrane proteins, playing critical roles in various signal transduction mechanisms. RLKs are large group in higher plants, there are about 610 RLKs in model plants Arabidopsis and rice, respectively [6, 7]. A typical RLK contains an extracellular receptor, a transmembrane domain and an intracellular Ser/Thr kinase domain[6]. Although roles for most RLKs are unknown, many RLKs were reported to regulate plant physiological processes, including development, hormone perception and defense responses [8].

Cysteine-rich RLKs (CRKs), a prominent subfamily of RLKs, is predicted to be transmembrane receptor proteins. CRKs are characterized by the presence of one to four copies of domain 26 of unknown function (DUF26), a C-X8-C-X2-C motif in the extracellular receptor region in the $\mathrm{N}$-terminus[9]. The conserved Cys residues may participate in the formation of the three-dimensional structure of the protein through disulfide bonds[10], which are known to mediate protein-protein interactions[11]. CRKs have been suggested to play important roles in the regulation of immune responses. Most of the CRKs in Arabidopsis are induced by pathogen attack and application of SA at the transcriptional level $[12,13]$. 
Some CRKs has been functionally characterized in the response to pathogen infection. For example, CRK45 acted as a positive regulator in Arabidopsis disease resistance. CRK45 overexpression enhanced resistance to Pseudomonas syringae, whereas the crk45 mutant was more sensitive to $P$. syringae[13] . Overexpression of CRK4, CRK6 and CRK36 in Arabidopsis increased disease resistance to the bacterial pathogen Pst DC3000 through a positive role in the PTI response by association with the FLS2 [14]. Intriguingly, recent studies revealed that some CRKs form a receptor complex with RLKs and play roles in transducing signals in the plant immune. For example, increased expression of CRK28 in Arabidopsis enhanced ROS burst and disease resistance toward $P$. syringae. CRK28 associated with FLS2/BAK1 immune complex as well as associated with the closely related CRK29 [15]. However, whether members of this large CRK subfamily in pepper regulate defense responses are unknown.

Here, we report the identification of a CRK coding genes CaCRK5 that function as a positive regulator in the pepper response to $R$. solanacearum infection. Suppression of CaCRK5 in pepper plants via virusinduced gene silencing (VIGS) increased susceptibility to pathogen. Overexpression of CaCRK5 in tobacco enhanced resistance to pathogen. In addition, CaCRK5 was targeted and up-regulated by CaHDZ27. Lastly, we demonstrate that CaCRK5 could interact with its homologous member CaCRK6 in the plasma membrane.

\section{Methods}

\section{Plant materials and Pathogen inoculation}

Pepper (Capsicum annuum cultivar L. cv. Fj8) was obtained from the pepper breeding group at Fujian Agriculture and Forestry University (FAFU), it exhibted medium-resistance to $R$. solanacearum that was identified by Prof. Shuilin He at FAFU in China. Pepper and tobacco (Nicotiana benthamiana) plants were grown in plastic pots containing steam-sterilized soil at $26^{\circ} \mathrm{C}$ with a long-day photoperiod $(16 \mathrm{~h} \mathrm{light/8 \textrm {h }}$ dark) with $60 \%$ relative humidity.

Virulent strains FJC100301 of $R$. solanacearum was used in this study. The $R$. solanacearum strain was grown in PSA medium. Bacteria cell solution was harvested with sterile $10 \mathrm{mM} \mathrm{MgCl}_{2}$ and re-suspended to $1.0 \times 10^{8} \mathrm{CFU} / \mathrm{mL}$. For pepper inoculation, seedlings were irrigated by cutting the roots and pouring 30 $\mathrm{mL}$ bacterial inoculum into each pot. For leaf inoculation, the leaf was infiltrated with $10 \mu \mathrm{L}$ of the $R$. solanacearum suspension using a syringe without a needle. For each plant, a disease index on a scale of 0 to 4 was calculated as described previously.

\section{Subcellular Localization}

For the subcellular localization assay, CaCRK5 were cloned into pMDC83 vector, resulting in 35S:CaCRK5GFP construct. The plasmids were transformed into A. tumefaciens GV1301. The transient expression assays were conducted as described previously [44]. Fluorescence of GFP in the transformed leaves was imaged using a confocal laser scanning microscope (TCSSP8; Leica, Solms, Germany). 


\section{Virus-Induced Gene Silencing}

The tobacco rattle virus (TRV)-based virus-induced gene silencing system was used for gene silencing in pepper. To achieve CaCRK5 specific silencing, about 200 bp DNA fragment in their 3'-untranslated region were PCR amplified and cloned into TRV2 to generate TRV2:CaCRK5. A. tumefaciens GV3101 carrying TRV1 with TRV2:CaCRK5 was co-infiltrated into the fully expanded cotyledons of pepper plants. Then, the plants were incubated at $16^{\circ} \mathrm{C}$ for $56 \mathrm{~h}$, and grown at $26^{\circ} \mathrm{C}$. The experiments were performed with at least 50 plants per treatment and repeated three times. The rate of the disease index was calculated as previously described[44].

\section{RNA extraction and quantitative real-time RT-PCR}

Total RNA was extracted with TRIzol Reagent (Invitrogen, Carlsbad, CA, USA). First-strand cDNAs were synthesized using One Step PrimeScript TM cDNA Synthesis Kit (TaKaRa, Shigo, Japan). The real-time RTPCR analysis was performed using a Bio-Rad real-time PCR system (Foster City, CA, USA.) and the SYBR Premix ExTaq II system (Takara). The relative expression of the CaCRK5 and defense-related genes were calculated with the $2^{-\Delta \Delta C t}$ method. CaActin or NtActin served as the reference gene to normalize the total amount of cDNA in each reaction. The primers were listed in Additional file 3.

\section{Yeast two-hybrid analysis}

The pGADT7 vector was used for GAL4 AD, and the pGBKT7 vector was used for GAL4 BD. ORFs of CaCRK5 and CaCRK6 were amplified and cloned into PGADT7 to generate constructs designated ADCaCRK5 and AD-CaCRK6. The cDNAs of CaCRK5 and CaCRK6 were amplified and cloned into pGBKT7 to generate constructs designated BD-CaCRK5 and BD-CaCRK6. The corresponding AD and BD plasmids were co-transformed into $\mathrm{Y} 2 \mathrm{H}$ Gold yeast strain. Their interaction was identified by spot assay. The transformants were tested on SD (Synthetic Dextrose) screening medium. The plates were incubated at $30^{\circ} \mathrm{C}$ till the formation of colonies.

\section{Bimolecular fluorescence complementation (BiFC) assay}

To generate BiFC constructs, CaCRK5 and CaCRK6 full length cDNAs were cloned into puc-SPYNE ${ }^{\mathrm{GW}}$ and puc-SPYCE ${ }^{\mathrm{GW}}$ vectors, which contain the $\mathrm{N}$-terminal or $\mathrm{C}$-terminal fragment of YFP (nYFP or cYFP, respectively). For transient expression, $A$. tumefaciens strain GV3101 carrying each construct was used to infiltrate leaves of 5-week-old $N$. benthamiana plants. For microscopic analysis, lower epidermis cells were observed using a confocal laser scanning microscope

\section{Tobacco transformation}

Transgenic tobacco plants were generated by using $A$. tumefaciens mediated tobacco leaf disc transformation [45]. The CaCRK5 coding region was cloned into pK7WG2 vector to get 35S:CRK5 constructs. Two constructs were introduced into A. tumefaciens GV3101 and used to transform $N$. 
benthamiana. Transformed seed stock was selected for kanamycin resistance by planting seeds on Murashige and Skoog agar plates containing $50 \mu \mathrm{g}$ of kanamycin per milliliter.

\section{Histochemical staining}

Histochemical staining was performed as previously described [44]. The infiltrated leaves were stained with trypan blue to visualize the cell death response and DAB solution for $\mathrm{H}_{2} \mathrm{O}_{2}$ detection.

\section{Electrophoretic mobility shift assay (EMSA)}

Expression of the CaHDZ27-GST fusion protein was induced in transformed E. coli BL21 cells, by adding IPTG (isopropyl $\beta$-D-1-thiogalactopyranoside, $0.05 \mathrm{mM}$ ) at $37^{\circ} \mathrm{C}$ for $4 \mathrm{~h}$. The recombinant proteins were extracted from the cells and purified using BeaverBeads ${ }^{\mathrm{TM}} \mathrm{GSH}$ protein purified kit according to manufacturer's instructions.

The EMSA was performed as described previously[46]. Cy5-labelled DNA fragments were synthesized and used as probes, while unlabeled DNA of the same sequence was used as a competitor.

\section{Chromatin immunoprecipitation (ChIP)-qPCR analysis}

ChIP assay was performed using the method as described previously[44]. Chromatin was extracted from pepper leaves infiltrated with 35S:HA-CaHDZ27 and cross-linked with $1 \%$ formaldehyde $(\mathrm{v} / \mathrm{v})$. The chromatin was broken into fragments by probe sonicator to an average length of 300 to $500 \mathrm{bps}$. The fragmented DNA was immunoprecipitated with anti-HA antibody. Enrichment of DNA samples was analyzed by quantitative real-time PCR.

\section{Results}

\section{CaCRK5 encodes a pathogen induced receptor-like kinase of the arginine aspartate (RD) family}

In CDNA-AFLP experiments designed to isolate genes involved in the pepper resistance to the infection of $R$. solanacearum, a partial CDNA fragment of CaCRK5 was obtained. As its expression was significantly upregulated after inoculation of $R$. solanacearum, we decided to study the function of this gene further. CaCRK5 cDNA clones were isolated from a cDNA library made from $R$. solanacearum inoculated leaves of pepper inbred line CM334. The proteins deduced from the CDNA clones of CaCRK5 contained 669 residues. SMART (http://smart.embl-heidelberg.de/) analysis of the domain architecture showed that their primary structures were composed of two cysteine-rich DUF26 domains, a transmembrane region and a serine/threonine kinase domain , and therefore CaCRK5 belongs to the family of cysteine-rich kinases [10]. In addition, CaCRK5 contains a conserved arginine-aspartic (RD) motif in the protein kinase domain (Fig.1). For most RD kinases, the phosphorylation in the activation loop is crucial for triggering kinase activity which usually displays phosphorylation/autophosphorylation ability. Non-RD kinases usually exhibit lower kinase activities because of the lack of activation loop autophosphorylation. RD and non-RD kinases often cooperate to control innate immune signaling in plants [16-19]. 


\section{Genome-wide identification of CRK family in pepper}

In the start of this work, we evaluate the CRK gene family in the pepper genome, Hidden Markov Model (HMM) profile of the Stress-antifung domain (PF01657) was used to search the pepper genome database PGP (http://peppergenome.snu.ac.kr/). The Arabidopsis CRK gene family sequences [10] were also used as query sequences to search against the PGP and the NCBI database. All of them were further subjected to domain analysis using NCBI-CDD (https://www.ncbi.nlm.nih.gov/Structure/cdd/wrpsb.cgi) and SMART, to ensure the presence of three important domains for typical CRK proteins including stressantifung domains, transmembrane domain, and kinase domain. A total of 27 CRK genes (CaCRKs) were identified in the pepper cultivar CM334 genome, which were numbered from CaCRK1 to CaCRK27 according to their localization on chromosomes. Except for CaCRK13/24/25 with only one DUF26 domain, most CRKs contained two DUF26 domains. The length of CaCRK proteins varied from 332 to $1120 \mathrm{aa}$, and molecular weights from $36.76 \mathrm{kDa}$ to $128.25 \mathrm{kDa}$. The predicted isoelectric points of CaCRK ranged from 5.53 to 9.37 (Additional file 1 ).

Chromosome physical localization analysis revealed that 27 CaCRKs were distributed on 7 of the 12 chromosomes in pepper. Chromosomes 2 contained the largest number of CRKs with 9 genes (34.6\%), chromosomes 12 had only 1 gene. Gene clustering was the most common feature of the CRK genes distribution (Fig. 2a). Phylogenetic analysis was performed using the full length protein sequences of CRK from pepper, and an unrooted phylogenetic tree constructed by the neighbor-joining (NJ) method. Multiple CRKs were present in tandem repeat on the same chromosome (e.g. CaCRK1-7, CaCRK8/9, CaCRK10/11, CaCRK13-15 and CaCRK24/25) (Fig. 2b). These results suggested that the expansion of CRKs in pepper may be due to tandem duplication. This distribution and physical clustering pattern is consistent with CRKs in Arabidopsis[15] and soybean[20].

\section{Expression of CaCRK5 response to infection of $R$. solanacearum and treatment with exogenous SA, MeJA and ETH}

To characterize the expression pattern of CaCRK5 in detail, quantitative real-time PCR (qRT-PCR) analyses were performed. 6-week-old pepper plants were leaf-inoculated with $R$. solanacearum. CaCRK5 was rapidly up-regulated over time after inoculation of $R$. solanacearum, and the highest level of expression was observed at $12 \mathrm{~h}$ post inoculation (hpi) with about 12.4-fold of that in control plant (Fig. 3a). Next, we investigated the expression of CaCRK5 treated with the defense-related signaling molecules salicylic acid $(\mathrm{SA})$, jasmonic acid (JA) and ethylene (ET). The analysis showed the expression of CaCRK5 was increased after SA treatment and reached a peak at $48 \mathrm{~h}$, and no significant change was observed after methy jasmonate (MeJA) or ethephon (ETH) treatment (Fig. 3b). These results supported that CaCRK5 was involved in pepper defense against $R$. solanacearum invasion.

\section{Subcellular localization of CRK5}

Since CaCRK5 encoded a potential transmembrane domain, it was predicted to localize to plasma membrane. To test the hypothesis, CaCRK5 was fused with green fluorescent protein (GFP) under the 
control of the CaMV 35S promoter. CBL1n protein, which is known to be localized to the plasma membrane [21], was fused with red fluorescent protein (RFP). 35S:GFP, 35S:CaCRK5-GFP were transiently co-expressed with 35S:CBL1n-RFP in leaf epidermal cells of $N$. benthamiana. As shown in Fig.4, 35S:GFP construct served as a negative control, and green fluorescence was ubiquitously distributed throughout the cell. CaCRK5-GFP green fluorescence overlapped very closely with the red fluorescence in the plasma membrane suggesting that they are localized on the plasma membrane in the plant cell.

\section{Silencing of CaCRK5 in pepper plants increases susceptibility to $R$. solanacearum infection}

To assess the role of CaCRK5 in the interaction between pepper and $R$. solanacearum, loss-of-function experiments in pepper seedlings by virus-induced gene silencing (VIGS) were performed [22]. As shown in Additional file 2, photobleaching was observed in newly emerged true leaves of plants infiltrated with Agrobacterium carrying CaPDS, indicating that the VIGS system worked efficiently. qRT-PCR analysis showed that expression of CaCRK5 in pepper leaves was significantly down-regulated during $R$. solanacearum infection in VIGS plants (Fig. 5a), indicating that CaCRK5 were efficiently silenced.

CaCRK5 silenced and control (TRV:00) pepper plants were subjected to $R$. solanacearum challenge. Phenotypic analysis indicated that CaCRK5 silenced pepper plants showed more severe disease symptoms than control plants after $R$. solanacearum infection (Fig. $5 b$ ). From 6 days post inoculation with $R$. solanacearum, CRK5 silenced pepper plants exhibited significant delay of wilt symptom. The disease index of CaCRK5 silenced plants was significantly increased, compared to the control plants (Fig. 5c). To address whether silencing of CaCRK5 affect growth of $R$. solanacearum, the bacterial population was determined. As shown in Fig. $5 \mathrm{~d}$, growth of $R$. solanacearum was significantly enhanced in CaCRK5 silenced plants 3 day post leaf-inocualtion, compared with control plants. Based on these observations, we believed that CaCRK5 participate defense responses in pepper.

Next, we investigated the cell death and oxidative burst in CaCRK5 silenced and the control leaves. Trypan blue and DAB staining confirmed that hypersensitive cell death and $\mathrm{H}_{2} \mathrm{O}_{2}$ accumulation were significantly reduced in CaCRK5 silenced leaves $48 \mathrm{~h}$ after inoculation with $R$. solanacearum (Fig. $5 \mathrm{e}$ ), indicating that $C a C R K 5$ play pivotal roles in early defense response associated with cell death during $R$. solanacearum infection. We further determined the effects of CaCRK5 silencing on the expression of defense related genes in pepper during $R$. solanacearum infection. qRT-PCR analyses showed that CaCRK5 silencing in pepper leaves significantly attenuated expression of defense related genes, including CaNPR1[23], CaSAR8.2[24], CaDEF1[25] and CaACO1[26], during R. solanacearum infection (Fig. 5f).

\section{Overexpression of CaCRK5 in tobacco reduces susceptibility to $R$. solanacearum infection}

A gain-of-function approach was also employed to study the function of CaCRK5 in the defense response. Due to technical difficulties, we used the tobacco plant (Nicotiana benthamiana), which is also a host for $R$. solanacearum. At least 10 transgenic tobacco lines were obtained and confirmed by kanamycin resistance analysis. Two $\mathrm{T}_{3} C a C R K 5$ overexpressed lines exhibited constitutive expression (Fig. 6a), and were used in subsequent experiments. 
Wild-type and transgenic plants (L3 and L7) at 4 weeks old were root-inoculated with $R$. solanacearum. The effect of CaCRK5 overexpression on development of bacterial wilt disease was observed. As shown in Fig. $6 \mathrm{~b}$, the CaCRK5 overexpressed plants showed much weaker symptoms at $12 \mathrm{~d}$ post-inoculation, compared with the wild-type. The wild-type plants showed wilt symptom from 6 day after inoculation and completely died $14 \mathrm{~d}$ after inoculation with $R$. solanacearum. The CaCRK5 overexpressing plants were observed significant delay of wilt symptom compared to wild-type plants (Fig. 6c), showing that overexpression of CaCRK5 conferred increased disease tolerance to $R$. solanacearum in tobacco. In addition, we also checked the expression of defense related genes, including NtPR2, NtPR3, NtHSR201 and NtHSR505. qRT-PCR analysis indicated that expressions of these tested defense related genes were increased in CaCRK5 overexpressed plants compared with wild-type plants, during the inoculation of $R$. solanacearum (Fig. 6d). Collectively, these data suggested that CaCRK5 overexpression enhanced defense responses against $R$. solanacearum infection in tobacco.

\section{CaCRK5 is directly regulated by transcription factor CaHDZ27}

To better understand the regulatory mechanism of CaCRK5, a 2000-bp promoter region upstream of the CaCRK5 coding sequence was identified. Sequence analysis using the PlantCARE database (http://bioinformatics.psb.ugent.be/webtools/plantcare/html/) suggested that the cis-elements in the promoter of CaCRK5 included two TCA-element involved in salicylic acid responsiveness, five binding sites for MYB transcription factor, and one binding sites for MYC. In addition, a known binding site (CAATTATTG) for the HD-Zip subfamily I member CaHDZ27 was located between positions -625 and -617. As CaHDZ27 also regulate the defense in pepper against $R$. solanacearum infecction[27], we speculated that CaCRK5 might be targeted by CaHDZ27.

An electrophoretic mobility shift assay (EMSA) was conducted to access the interaction of CaHDZ27 with the CaCKR5 promoter, and CaHDZ27 was found to bind to the Cy5-labeled CaCKR5 promoter probe. Moreover, binding was gradually attenuated by increasing concentrations of unlabeled probe, indicating that CaHDZ27 binds specifically to the CAATTATTG in the CaCKR5 promoter in vivo (Fig. 7a). Chromatin immunoprecipitation (ChIP)-qPCR was conducted to confirm interaction of CaHDZ27 and the CaCKR5 promoter in vitro. The pepper leaves infiltrated with Agrobacterium strain GV3101 containing 35S:HACaHDZ27 or 35S:HA were harvested for ChIP assay. Chromatin from these pepper leaves was immunoprecipitated using anti-HA antibodies and enrichment of DNA sample was determined by qRTPCR. The result showed that CaHDZ27 was significantly enriched in the CaCKR5 promoter, and the enrichment was significantly enhanced by the inoculation of $R$. solanacearum (Fig. $7 \mathrm{~b}$ and c), suggesting that CaHDZ27 could bind to the CAATTATTG in the CaCKR5 promoter in vitro, and binding was increased by the $R$. solanacearum infection.

To further investigate the CaCKR5 regulated by CaHDZ27 in the transcriptional level, we transiently expressed 35S:CaHDZ27-GFP in pepper leaves, and 35S:GFP infiltrated leaves were used as a negative control. qRT-PCR analysis demonstrated that the transcript level of CaCKR5 was increased in 35S:CaHDZ27-GFP infiltrated leaves compared with the control(Fig. 8a). Using anti-GFP immune-blotting, 
CaHDZ27 was confirmed to be expressed in pepper leaves (Fig. 8b). We used a VIGS approach to silence CaHDZ27. The transcript level of CaCKR5 is reduced significantly in CaHDZ27-silenced plants compared with TRV:00 infiltrated plants, $48 \mathrm{~h}$ post the inoculation of $R$. solanacearum (Fig. $8 \mathrm{c}$ and d). These data suggested that the transcriptional expression of $C a C K R 5$ was positively regulated by CaHDZ27.

\section{CaCRK5 interacts with CaCRK6}

Previous studies indicated that plants employ immune receptor complex for sensing microbe-derived molecular patterns and effectors to trigger inducible immune defenses[28-31], and a few CRKs have been known to function in association with each other, such as AtCRK28/AtCRK29[15] and AtCRK39/AtCRK40[32]. Thus, we investigated the ability of CaCRK5 to heterodimerize with the closely related CaCRK6, which has $77.4 \%$ amino acid sequence identity to CaCRK5.

The cDNAs of CaCRK5 and CaCRK6 were cloned into the pGBKT7 and pGADT7 vectors separately, and generated DNA-binding domain (BD) and activation domain (AD) fusions. BD-CaCRK5/AD-CaCRK6 and BD-CaCRK6/AD-CaCRK5 transformant yeast cells can grow on SD/-Trp-Leu-His-Ade medium, as did positve control (pGBKT7-53/pGADT7-T). In contrast, no growth was observed in the negative controls. The result indicated that CaCRK5 interacts with CaCRK6 each other in yeast cells (Fig. 9a). To provide more evidence for the interaction between CaCRK5 and CaCRK6, we performed bimolecular fluorescence complementation (BiFC) assays. CaCRK5 and CaCRK6 were fused to the $\mathrm{N}$ - and $\mathrm{C}$-terminal ends of yellow fluorescent protein (YFP) to generate CaCRK5-nYFP/CaCRK6-cYFP and CaCRK5-cYFP/CaCRK6-nYFP, respectively. Then, these constructs were transiently co-expressed in Nicotiana benthamiana leaves. At 48h post infiltration, the YFP fluorescence signals were checked by by confocal microscopy. As showed in Fig. 9b, YFP fluorescence was oboserved at the plasma membrane in $N$. benthamiana leaves. These results indicate that CaCRK5 heterodimerizes with CaCRK6 at the plasma membrane of plant cells.

\section{Discussion}

\section{The tandem repeats contributed to the expansion of the CaCRK family in pepper}

CRKs are a subfamily of plant RLKs. In this study, 27 CaCRKs were identified in pepper genome; many CRKs were present in tandem repeat which made physical clusters at specific positions in the chromosome. Previous studies showed that a large proportion of the RLK gene family is found in tandem repeats [33-35]. Tandem repeats are enriched in stress-responsive genes [36-38]. The expansion and degree of tandem repeats are significantly correlated with the stress responsiveness of RLK genes[36]. The tandem repeats of CaCRKs in pepper may facilitate the stress adaptive evolution.

\section{CaCRK5 played a positve role in the pepper resistance against $R$. solanacearum}

Previous studies have shown that some CRK members including AtCRK29 in Arabidopsis were induced in response to treatment with pathogens, SA and flg22 [15,39,40], suggesting a role for these CRKs in plant immunity. The CaCRK5 closest homologue in Arabidopsis is AtCRK29 protein with $41.6 \%$ identity to 
CaCRK5. In the study, expressions of CaCRK5 were increased over time after inoculation of $R$. solanacearum, with high levels of gene expression at the early stage of infection. CaCRK5 was strongly up-regulated after treatment with SA, and no significant change of $C R K 5$ was observed treated with MeJA and ETH.

Based on the subcellular localizations of GFP fusions, CaCRK5 are probably targeted to the plasma membrane. Several CRK proteins also have been reported to be localized in the plasma membrane, including CRK4, CRK6, and CRK36 in Arabidopsis [14] and GbCRK18 in cotton[41]. The TRV-based VIGS system was used to investigate the function of CaCRK5 in the response to $R$. solanacearum infection. Expression of CaCRK5 was significantly down-regulated during $R$. solanacearum infection in pepper leaves in which CaCRK5 was silenced. CaCRK5 silenced pepper plants showed an increased susceptibility to infection by $R$. solanacearum, pepper leaves significantly compromised $\mathrm{H} 2 \mathrm{O} 2$ accumulation and cell death. These findings suggested that CaCRK5 may function as a positive regulator in pepper resistance against $R$. solanacearum infection. Furthermore, transient expression of CaCRK5 in pepper leaves resulted in HR-like cell death, which was accompanied by accumulation of ROS. Consistently, CaCRK5 overexpression tobacco plants exhibited enhanced resistance against infection by R. solanacearum.

\section{CaCRK5 was transcriptionally regulated directly by CaHDZ27}

HD-Zip transcription factors can be divided into four subfamilies (HD-Zip I-IV) based on their sequence similarities[42], and HD-Zip I proteins were reported to play roles in biotic and abiotic stress responses[43]. CaHDZ27, a HD-Zip I member, acts a positive regulator in pepper response to $R$. solanacearum infection[27]. Here we showed that CaHDZ27 acts as a positive regulator of CaCRK5, CaHDZ27 binds to a 9-bp CAATTATTG motif of the CaCRK5 promoter revealed by EMSA and ChIP-qPCR. Expression of CaCRK5 is increased in CaHDZ27 transit expressed pepper leaves, and decreased in CaHDZ27 silenced pepper plants with the inoculation of $R$. solanacearum. Moreover, CaCRK5 play a positive role in pepper resistance agianst $R$. solanacearum infection, consistent with the role of CaHDZ27. These results suggest that CaCRK5 and CaHDZ27 coordinate to regulate the pepper defense against $R$. solanacearum infection.

\section{CaCRK5 heterodimerizes with CaCRK6}

Yeast two-hybrid and bimolecular fluorescence complementation analyses indicated that CaCRK5 heterodimerizes with CaCRK6 in vivo in both yeast and plant cells. Recent evidence suggest that pattern recognition receptors (PRRs) enable the rapid recruitment of other RLKs, including CRKs, to enhance PRR signaling intensity $[15,39]$. CaCRK5 might act in concert with CaCRK6, and other RLKs in the pepper defense against $R$. solanacearum infection.

\section{Conclusions}


In summary, our data suggest that CaCRK5 may significantly contribute to immune defense against $R$. solanacearum in pepper and tobacco. VIGS in pepper plants and gain-of-function analyses of CaCRK5 in tobacco, revealed that CaCRK5 positively regulates plant immune responses. In addition, CaHDZ27 promotes CaCRK5 expression by directly binding to its promoter. We propose that the pepper defense response mediated by CaCRK5 could be enhanced by CaHDZ27.

\section{Abbreviations}

CRK: Cysteine-rich receptor-like kinases; VIGS: Virus-induced gene silencing; PRR: pattern recognition receptor; PAMP: pathogen-associated molecular patterns; PTI: PAMP-triggered immunity; HMM: Hidden Markov Model; R. solanacearum: Ralstonia solanacearum; hpi: hours post inoculation; SA: Salicylic acid; JA: Jasmonic acid; ET: ethylene.

\section{Declarations}

\section{Acknowledgements}

We thank Mark D. Curtis for kindly providing the Gateway destination vectors and Dr. S. P. Dinesh-Kumar (Yale University) for the PTRV1 and pTRV2 vectors.

\section{Author contributions}

SM and SH conceived and designed the research. SM, FG, TZ, WH and QM organized and performed the experiments. SM and FG analyzed the data. SM and SH wrote the manuscript. All authors read and approved the final manuscript.

\section{Funding}

This work was supported by grants from the National Natural Science Foundation of China (31301254, $31372061,31601761,31401312$ and 31260482) and Natural Science Foundation of Fujian Province (2018J01616). The funding bodies have no roles in the design of research, the data analysis, and the manuscript preparation.

\section{Availability of data and materials}

All data generated or analysed during this study are included in this published article and its supplementary information files.

\section{Ethics approval and consent to participate}

Not applicable.

\section{Consent for publish}


Not applicable.

Competing interests

The authors have no conflicts of interest to declare.

\section{References}

1. Bhattarai D, Worku T, Dad R, Rehman ZU, Gong X, Zhang S: Mechanism of pattern recognition receptors (PRRs) and host pathogen interplay in bovine mastitis. Microb Pathog 2018, 120:64-70.

2. Wang L, Albert M, Einig E, Furst U, Krust D, Felix G: The pattern-recognition receptor CORE of Solanaceae detects bacterial cold-shock protein. Nat Plants 2016, 2:16185.

3. Tang D, Wang G, Zhou JM: Receptor kinases in plant-pathogen interactions: More than pattern recognition. Plant Cell 2017, 29(4):618-637.

4. Gomez-Gomez L, Boller T: FLS2: an LRR receptor-like kinase involved in the perception of the bacterial elicitor flagellin in Arabidopsis. Mol Cell 2000, 5(6):1003-1011.

5. Zipfel C, Kunze G, Chinchilla D, Caniard A, Jones JD, Boller T, Felix G: Perception of the bacterial PAMP EF-Tu by the receptor EFR restricts Agrobacterium-mediated transformation. Cell 2006, 125(4):749-760.

6. Shiu SH, Bleecker AB: Receptor-like kinases from Arabidopsis form a monophyletic gene family related to animal receptor kinases. Proc Natl Acad Sci U S A 2001, 98(19):10763-10768.

7. Mazaheri M, Heckwolf M, Vaillancourt B, Gage JL, Burdo B, Heckwolf S, Barry K, Lipzen A, Ribeiro CB, Kono TJY et al: Genome-wide association analysis of stalk biomass and anatomical traits in maize. BMC Plant Biol 2019, 19(1):45.

8. Yuan N, Rai KM, Balasubramanian VK, Upadhyay SK, Luo H, Mendu V: Genome-wide identification and characterization of LRR-RLKs reveal functional conservation of the SIF subfamily in cotton (Gossypium hirsutum). BMC Plant Biol 2018, 18(1):185.

9. Wrzaczek M, Brosche M, Salojarvi J, Kangasjarvi S, Idanheimo N, Mersmann S, Robatzek S, Karpinski S, Karpinska B, Kangasjarvi J: Transcriptional regulation of the CRK/DUF26 group of receptor-like protein kinases by ozone and plant hormones in Arabidopsis. BMC Plant Biol 2010, 10:95.

10. Chen Z: A superfamily of proteins with novel cysteine-rich repeats. Plant Physio/ 2001, 126(2):473476.

11. Rayapuram C, Jensen MK, Maiser F, Shanir JV, Hornshoj H, Rung JH, Gregersen PL, Schweizer P, Collinge DB, Lyngkjaer MF: Regulation of basal resistance by a powdery mildew-induced cysteinerich receptor-like protein kinase in barley. Mol Plant Pathol 2012, 13(2):135-147.

12. Chen K, Du L, Chen Z: Sensitization of defense responses and activation of programmed cell death by a pathogen-induced receptor-like protein kinase in Arabidopsis. Plant Mol Biol 2003, 53(1-2):6174. 
13. Zhang X, Han X, Shi R, Yang G, Qi L, Wang R, Li G: Arabidopsis cysteine-rich receptor-like kinase 45 positively regulates disease resistance to Pseudomonas syringae. Plant Physiol Biochem 2013, 73:383-391.

14. Yeh YH, Chang YH, Huang PY, Huang JB, Zimmerli L: Enhanced Arabidopsis pattern-triggered immunity by overexpression of cysteine-rich receptor-like kinases. Front Plant Sci 2015, 6:322.

15. Yadeta KA, Elmore JM, Creer AY, Feng B, Franco JY, Rufian JS, He P, Phinney B, Coaker G: A Cysteinerich protein kinase associates with a membrane immune complex and the cysteine residues are required for cell death. Plant Physio/ 2017, 173(1):771-787.

16. de Oliveira LFV, Christoff AP, de Lima JC, de Ross BCF, Sachetto-Martins G, Margis-Pinheiro M, Margis R: The Wall-associated kinase gene family in rice genomes. Plant Sci 2014, 229:181-192.

17. Wu W, Wu Y, Gao Y, Li M, Yin H, Lv M, Zhao J, Li J, He K: Somatic embryogenesis receptor-like kinase 5 in the ecotype Landsberg erecta of Arabidopsis is a functional RD LRR-RLK in regulating brassinosteroid signaling and cell death control. Front Plant Sci 2015, 6:852.

18. Dardick C, Schwessinger B, Ronald P: Non-arginine-aspartate (non-RD) kinases are associated with innate immune receptors that recognize conserved microbial signatures. Curr Opin Plant Biol 2012, 15(4):358-366.

19. Dardick C, Ronald P: Plant and animal pathogen recognition receptors signal through non-RD kinases. PLoS Pathog 2006, 2(1):e2.

20. Delgado-Cerrone L, Alvarez A, Mena E, Ponce de Leon I, Montesano M: Genome-wide analysis of the soybean CRK-family and transcriptional regulation by biotic stress signals triggering plant immunity. PLoS One 2018, 13(11):e0207438.

21. Batistic O, Sorek N, Schultke S, Yalovsky S, Kudla J: Dual fatty acyl modification determines the localization and plasma membrane targeting of CBL/CIPK Ca2+ signaling complexes in Arabidopsis. Plant Cell 2008, 20(5):1346-1362.

22. Liu Y, Schiff M, Dinesh-Kumar SP: Virus-induced gene silencing in tomato. Plant J 2002, 31(6):777786.

23. Shen L, Liu Z, Yang S, Yang T, Liang J, Wen J, Liu Y, Li J, Shi L, Tang Q et al: Pepper CabZIP63 acts as a positive regulator during Ralstonia solanacearum or high temperature-high humidity challenge in a positive feedback loop with CaWRKY40. J Exp Bot 2016, 67(8):2439-2451.

24. Lee SC, Hwang BK: Identification of the pepper SAR8.2 gene as a molecular marker for pathogen infection, abiotic elicitors and environmental stresses in Capsicum annuum. Planta 2003, 216(3):387-396.

25. Choi HW, Lee BG, Kim NH, Park Y, Lim CW, Song HK, Hwang BK: A role for a menthone reductase in resistance against microbial pathogens in plants. Plant Physiol 2008, 148(1):383-401.

26. Cai H, Yang S, Yan Y, Xiao Z, Cheng J, Wu J, Qiu A, Lai Y, Mou S, Guan D et al: CaWRKY6 transcriptionally activates CaWRKY40, regulates Ralstonia solanacearum resistance, and confers high-temperature and high-humidity tolerance in pepper. J Exp Bot 2015, 66(11):3163-3174. 
27. Mou S, Liu Z, Gao F, Yang S, Su M, Shen L, Wu Y, He S: CaHDZ27, a Homeodomain-Leucine zipper I protein, positively regulates the resistance to Ralstonia solanacearum infection in pepper. Mol Plant Microbe Interact 2017, 30(12):960-973.

28. Saijo Y, Loo EP, Yasuda S: Pattern recognition receptors and signaling in plant-microbe interactions. Plant J 2018, 93(4):592-613.

29. Bohm H, Albert I, Fan L, Reinhard A, Nurnberger T: Immune receptor complexes at the plant cell surface. Current Opinion in Plant Biology 2014, 20:47-54.

30. Lin W, Li B, Lu D, Chen S, Zhu N, He P, Shan L: Tyrosine phosphorylation of protein kinase complex BAK1/BIK1 mediates Arabidopsis innate immunity. Proc Natl Acad Sci U S A 2014, 111(9):36323637.

31. Liu J, Chen S, Chen L, Zhou Q, Wang M, Feng D, Li JF, Wang J, Wang HB, Liu B: BIK1 cooperates with BAK1 to regulate constitutive immunity and cell death in Arabidopsis. J Integr Plant Biol 2017, 59(4):234-239.

32. Tanaka H, Osakabe Y, Katsura S, Mizuno S, Maruyama K, Kusakabe K, Mizoi J, Shinozaki K, Yamaguchi-Shinozaki K: Abiotic stress-inducible receptor-like kinases negatively control ABA signaling in Arabidopsis. Plant J 2012, 70(4):599-613.

33. Sun J, Li L, Wang P, Zhang S, Wu J: Genome-wide characterization, evolution, and expression analysis of the leucine-rich repeat receptor-like protein kinase (LRR-RLK) gene family in Rosaceae genomes. BMC Genomics 2017, 18(1):763.

34. Nazarian-Firouzabadi F, Joshi S, Xue H, Kushalappa AC: Genome-wide in silico identification of LysM-RLK genes in potato (Solanum tuberosum L.). Mol Biol Rep 2019, 46(5):5005-5017.

35. Wei Z, Wang J, Yang S, Song Y: Identification and expression analysis of the LRR-RLK gene family in tomato (Solanum lycopersicum) Heinz 1706. Genome 2015, 58(4):121-134.

36. Lehti-Shiu MD, Zou C, Hanada K, Shiu SH: Evolutionary history and stress regulation of plant receptor-like kinase/pelle genes. Plant Physiol 2009, 150(1):12-26.

37. Hanada K, Zou C, Lehti-Shiu MD, Shinozaki K, Shiu SH: Importance of lineage-specific expansion of plant tandem duplicates in the adaptive response to environmental stimuli. Plant Physio/2008, 148(2):993-1003.

38. Rizzon C, Ponger L, Gaut BS: Striking similarities in the genomic distribution of tandemly arrayed genes in Arabidopsis and rice. PLoS Comput Biol 2006, 2(9):e115.

39. Bourdais G, Burdiak P, Gauthier A, Nitsch L, Salojarvi J, Rayapuram C, Idanheimo N, Hunter K, Kimura $\mathrm{S}$, Merilo $\mathrm{E}$ et al: Large-Scale phenomics identifies primary and fine-tuning roles for CRKs in responses related to oxidative stress. PLoS Genet 2015, 11(7):e1005373.

40. Du L, Chen Z: Identification of genes encoding receptor-like protein kinases as possible targets of pathogen- and salicylic acid-induced WRKY DNA-binding proteins in Arabidopsis. Plant $\mathrm{J} 2000$, 24(6):837-847.

41. Li TG, Zhang DD, Zhou L, Kong ZQ, Hussaini AS, Wang D, Li JJ, Short DPG, Dhar N, Klosterman SJ et al: Genome-wide identification and functional analyses of the CRK gene family in cotton reveals 
GbCRK18 confers verticillium wilt resistance in Gossypium barbadense. Front Plant Sci 2018, 9:1266.

42. Ariel FD, Manavella PA, Dezar CA, Chan RL: The true story of the HD-Zip family. Trends Plant Sci 2007, 12(9):419-426.

43. Romani F, Ribone PA, Capella M, Miguel VN, Chan RL: A matter of quantity: Common features in the drought response of transgenic plants overexpressing HD-Zip I transcription factors. Plant Sci 2016, 251:139-154.

44. Mou S, Gao F, Shen L, Yang S, He W, Cheng W, Wu Y, He S: CaLRR-RLK1, a novel RD receptor-like kinase from Capsicum annuum and transcriptionally activated by CaHDZ27, act as positive regulator in Ralstonia solanacearum resistance. BMC Plant Bio/ 2019, 19(1):28.

45. Oh SK, Park JM, Joung YH, Lee S, Chung E, Kim SY, Yu SH, Choi D: A plant EPF-type zinc-finger protein, CaPIF1, involved in defence against pathogens. Mol Plant Pathol 2005, 6(3):269-285.

46. Shen L, Yang S, Yang F, Guan D, He S: CaCBL1 acts as a positive regulator in pepper response to Ralstonia solanacearum. Mol Plant Microbe Interact 2020, 33(7):945-957.

\section{Figures}

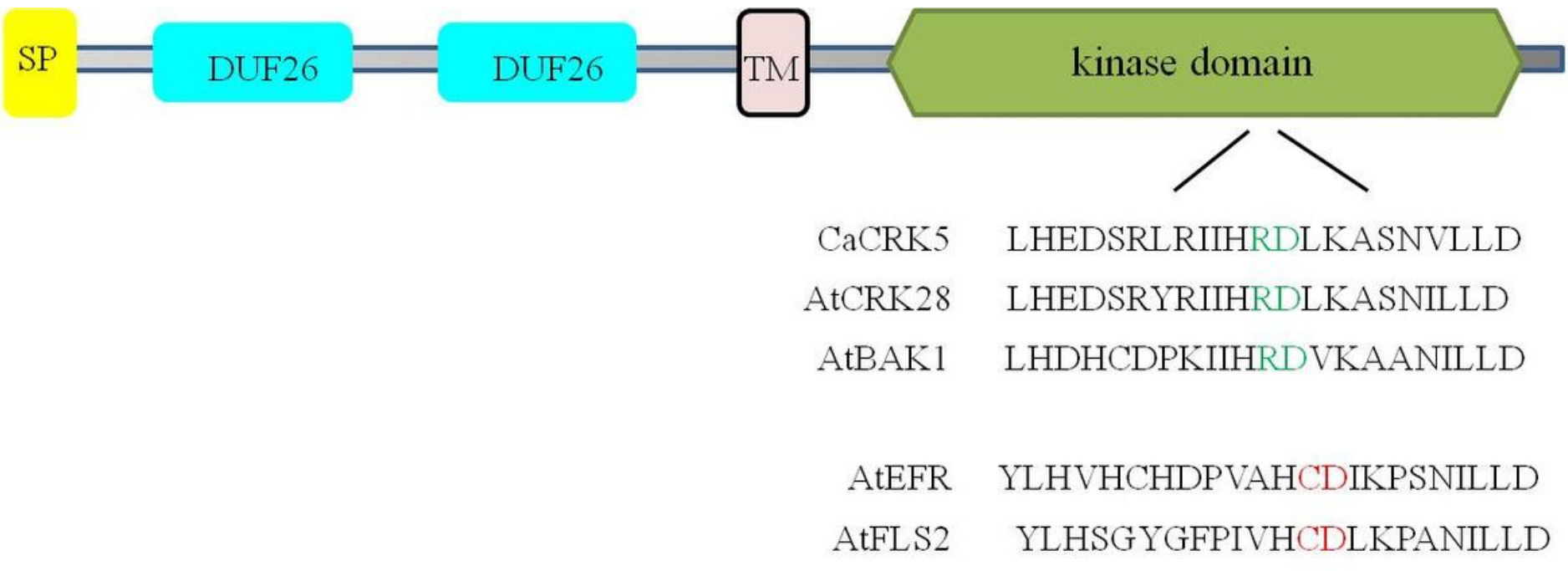

\section{Figure 1}

Schematic diagram of CaCRK5 protein domain architecture. It contains the signal peptide (SP), two domains 26 of unknown function (DUF26), transmembrane domain and kinase domain. The protein kinase domain harbors a RD motif represented by a green R and D. non-RD motif, which is often found in association with pattern recognition receptors such as AtEFR and AtFLS2, was represented by red $\mathrm{C}$ and D. 


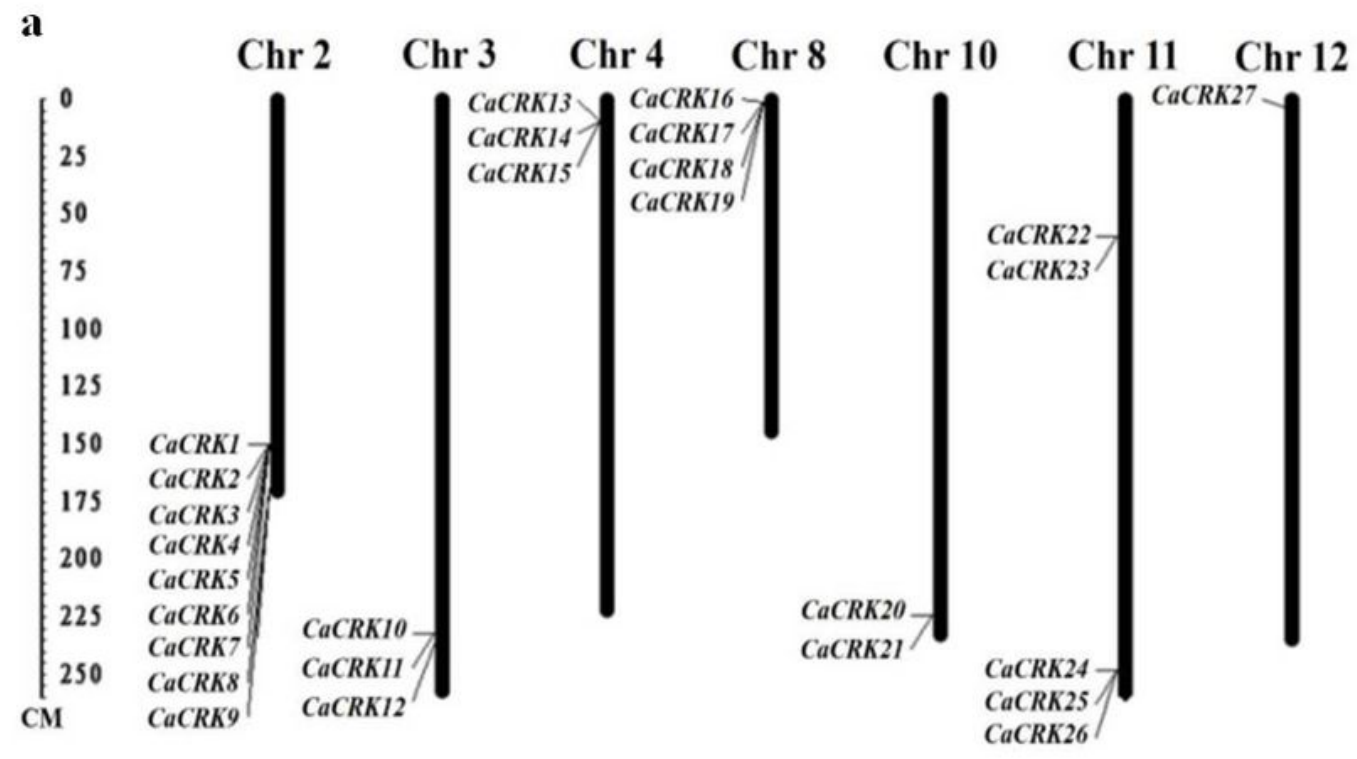

b

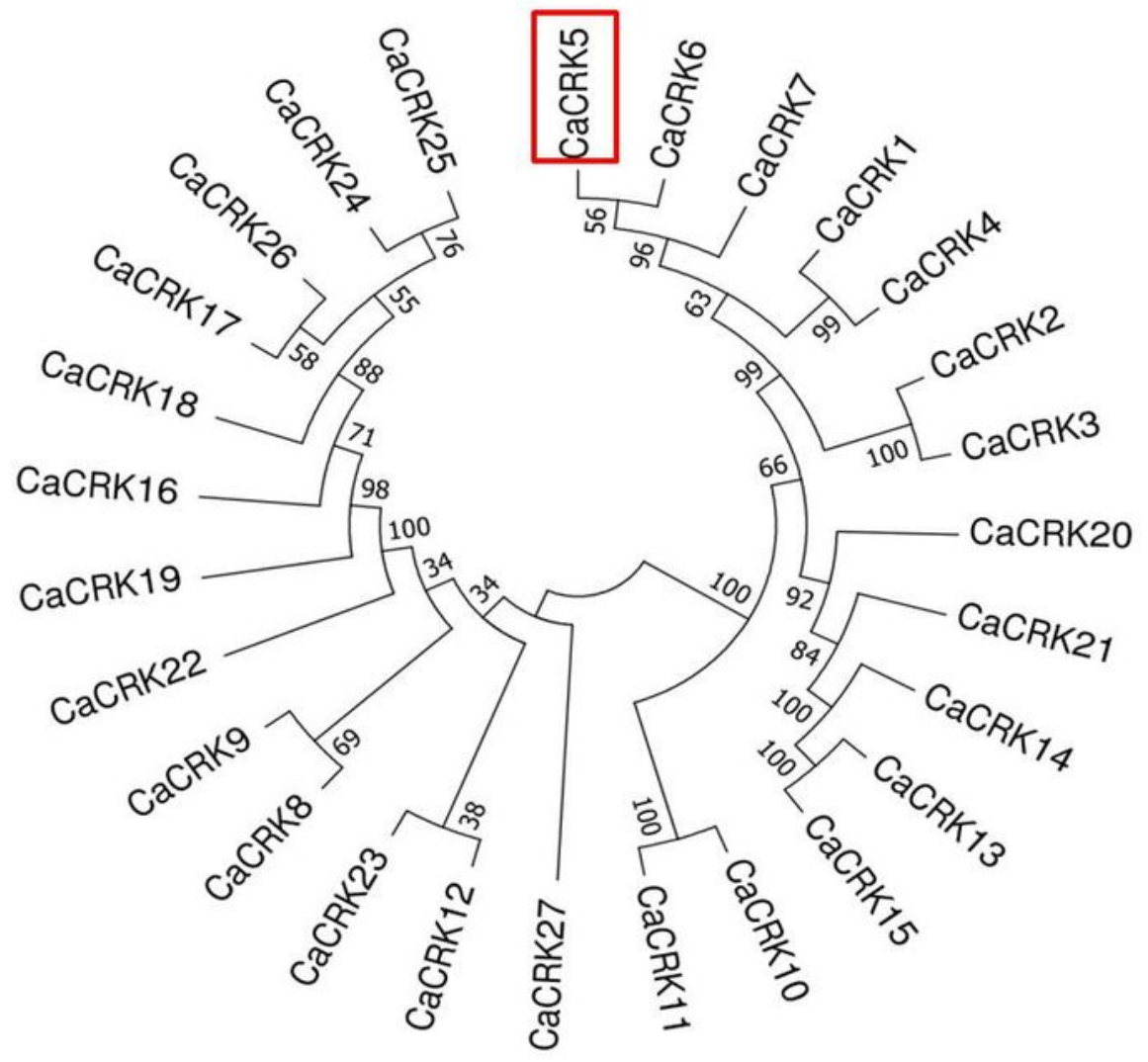

Figure 2

Chromosomal location and phylogenetic analysis of CRK gene family. a Physical genome distribution of CRK gene family in pepper. b Phylogenetic tree was generated based on the amino acid sequences of CRK genes in pepper using the neighbor-joining method in MEGA version 5.1. 
a

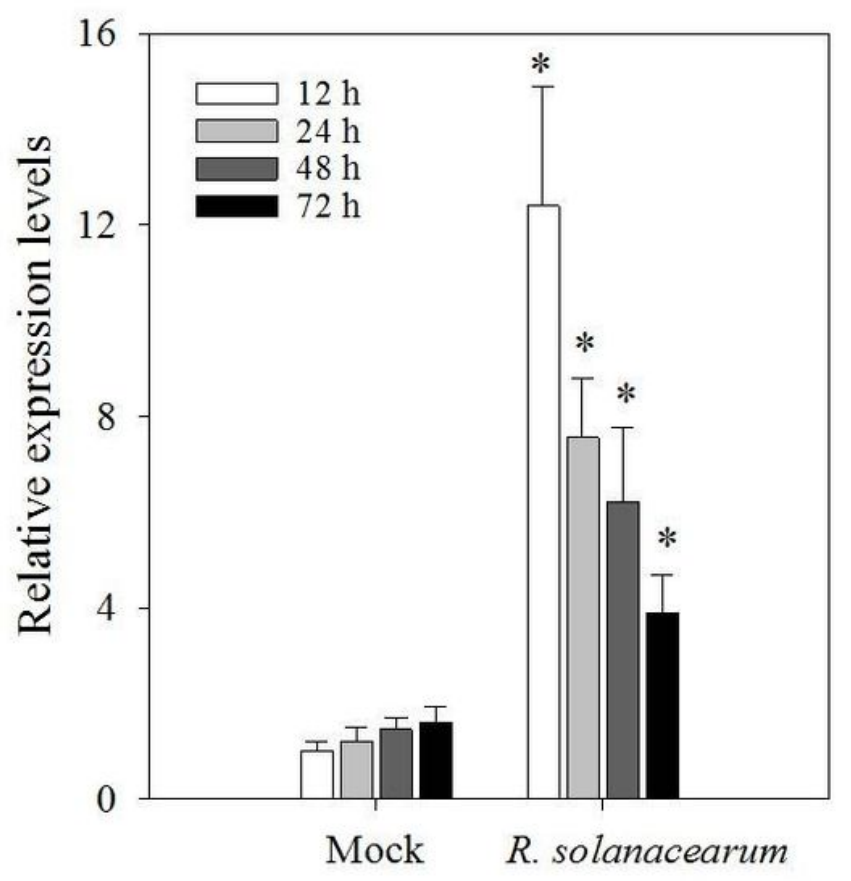

b

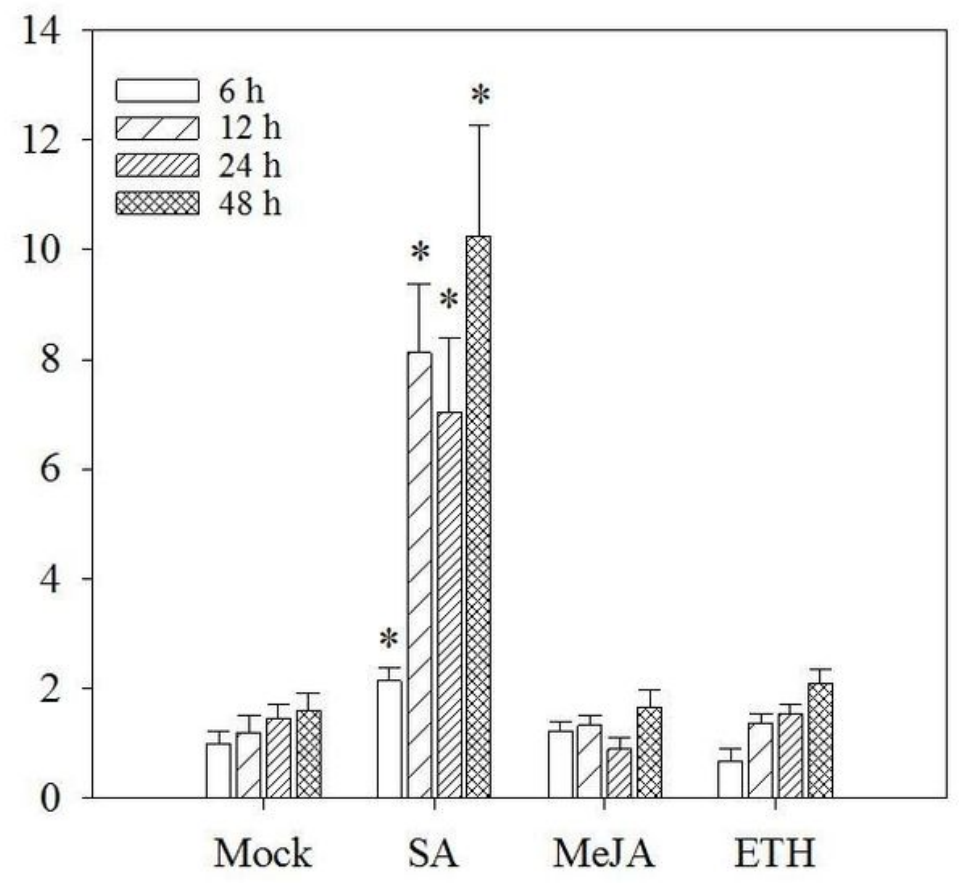

\section{Figure 3}

Expression patterns of CaCRK5 under different treatments. a Expression analysis of CaCRK5 in pepper after inoculation with R. solanacearum by qRT-PCR. b Expression analysis of CaCRK5 in pepper responding to SA, MeJA and ETH. Error bars represent the mean \pm SD of three independent replicates. Asterisks indicate significant differences as determined by Student's t-test $(P<0.05)$.

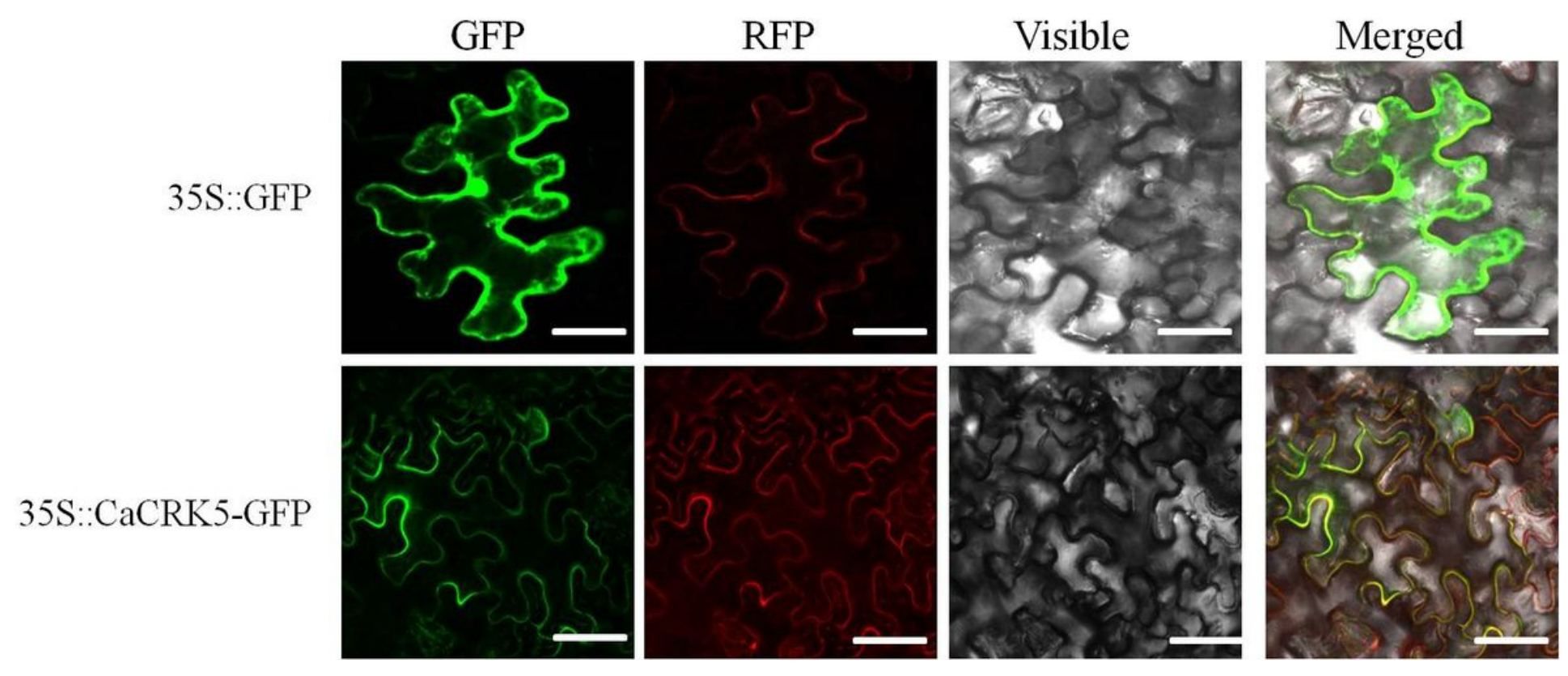

\section{Figure 4}

Subcellular localization of CaCRK5. Six-week-old tobacco leaves were infiltrated with Agrobacterium carrying 35S:GFP, 35S:CaCRK5-GFP and 35S:CBL1n-RFP. Samples were collected $48 \mathrm{~h}$ post inoculation. 
Fluorescence was examined using a confocal microscope. GFP, green fluorescent protein; RFP, red fluorescent protein. Bars, $50 \mu \mathrm{m}$.

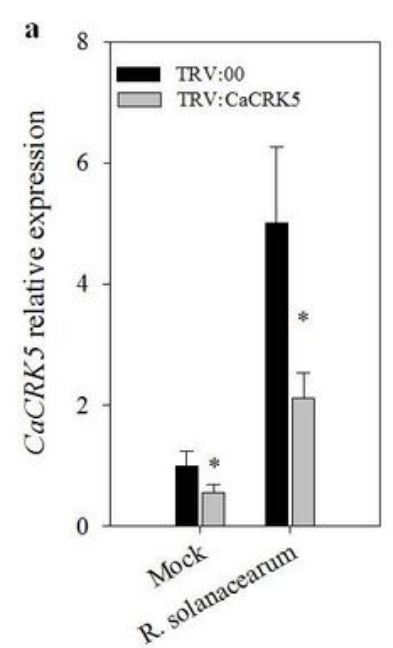

c

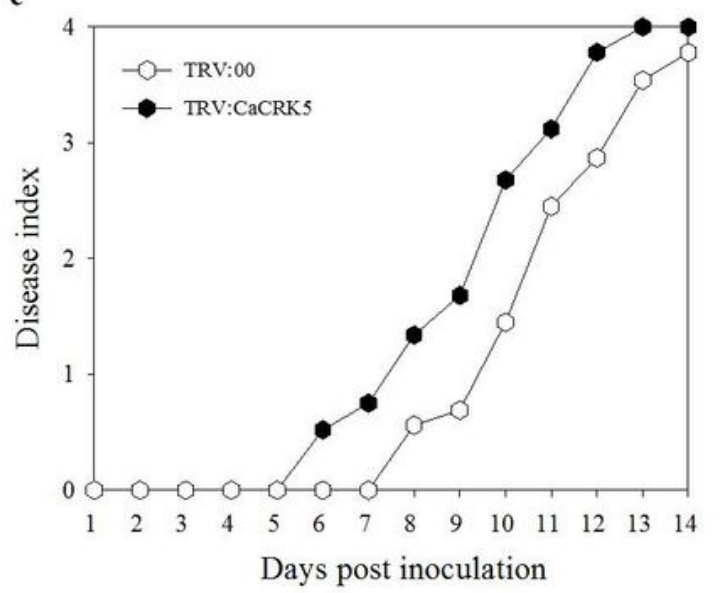

b

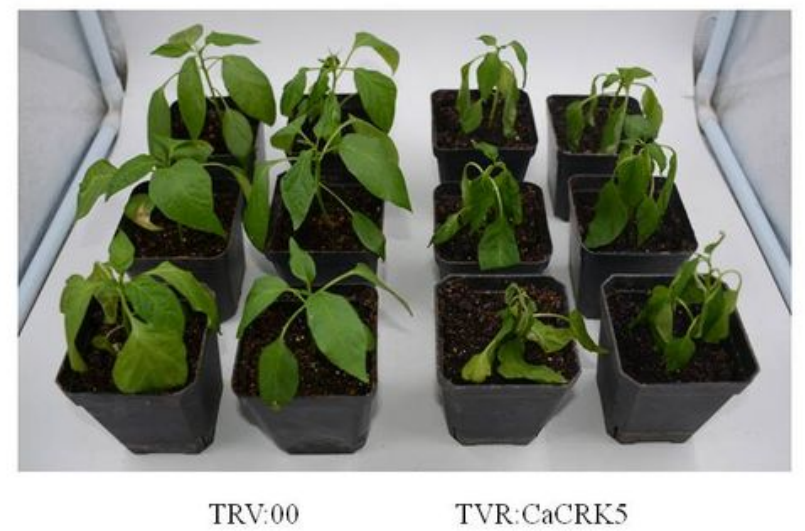

d

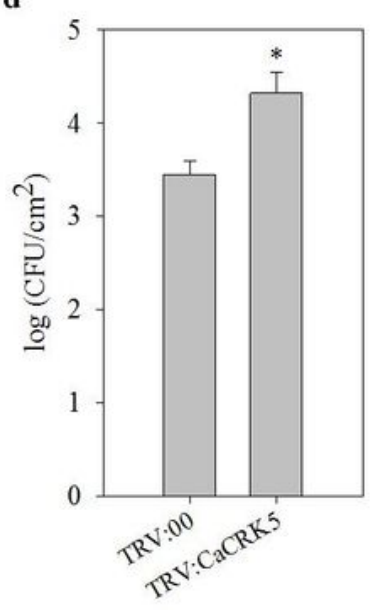

e

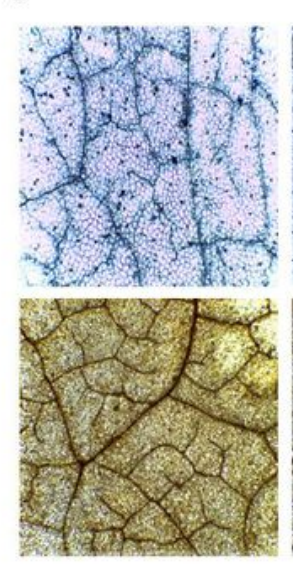

TRV:00

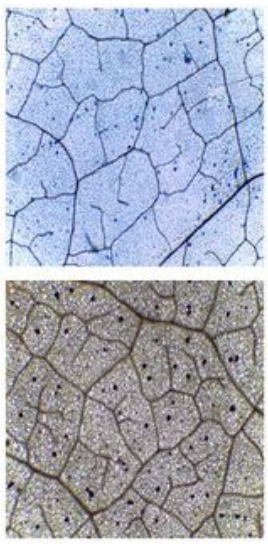

TVR:CaCRK5

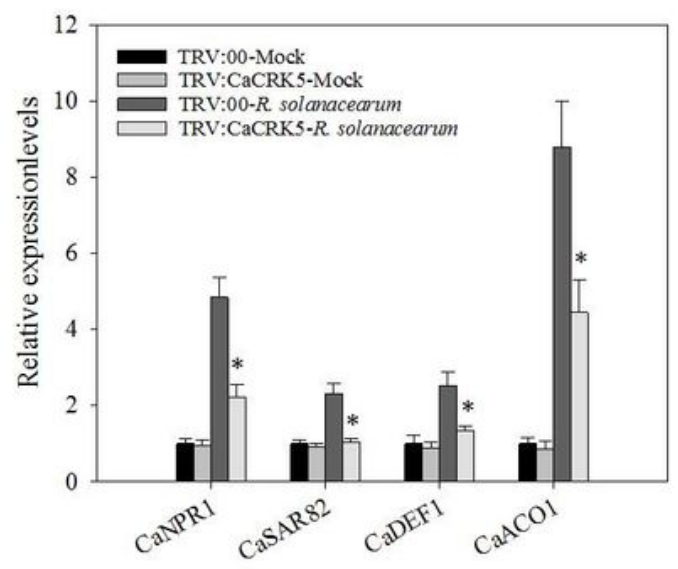

Figure 5

Silencing of CaCRK5 enhanced susceptibility to infection of R. solanacearum in pepper. a CaCRK5 was silenced by VIGS. The leaf samples from CaCRK5 VIGS pepper seedlings were collected to detect the expression of CaCRK5 by qRT-PCR. Error bars represent the mean \pm SD of three independent replicates. 
The experiments were repeated three times with similar results. b Phenotypes of control and VIGS pepper plants. After the VIGS was established, 4 week old plants were inoculated with R. solanacearum.

Photographs were taken at $10 \mathrm{~d}$ after R. solanacearum infection. c Rate of disease index on a scale of 0 to 4 was calculated in CaCRK5 silenced and control plants. $d$ Growth of R. solanacearum in leaves of TRV:CaCRK5 and plants at $3 \mathrm{~d}$ post leaf inoculation of R. solanacearum. The data are shown as means \pm $S D$ from three independent repeats $(n=3, *, P<0.05$, Student's t-test). e Visualization of diaminobenzidine (DAB) and trypan blue staining $48 \mathrm{~h}$ post leaf inoculation with $\mathrm{R}$. solanacearum. $\mathrm{f}$ The expression levels of defense related genes in control (TRV:00) and CaCRK5-silenced (TRV:CaCRK5) plants. Asterisks indicate statistically significant differences (Student's $t$ test, ${ }^{*} \mathrm{P}<0.05$ ).

a

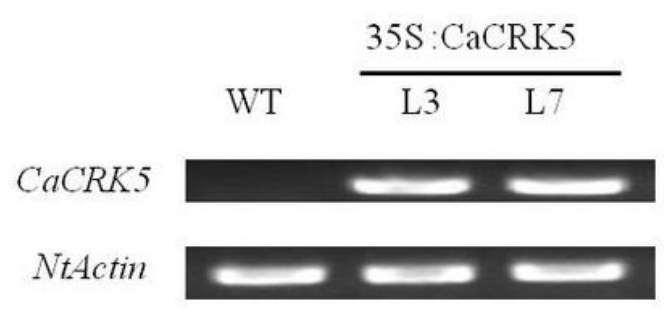

c

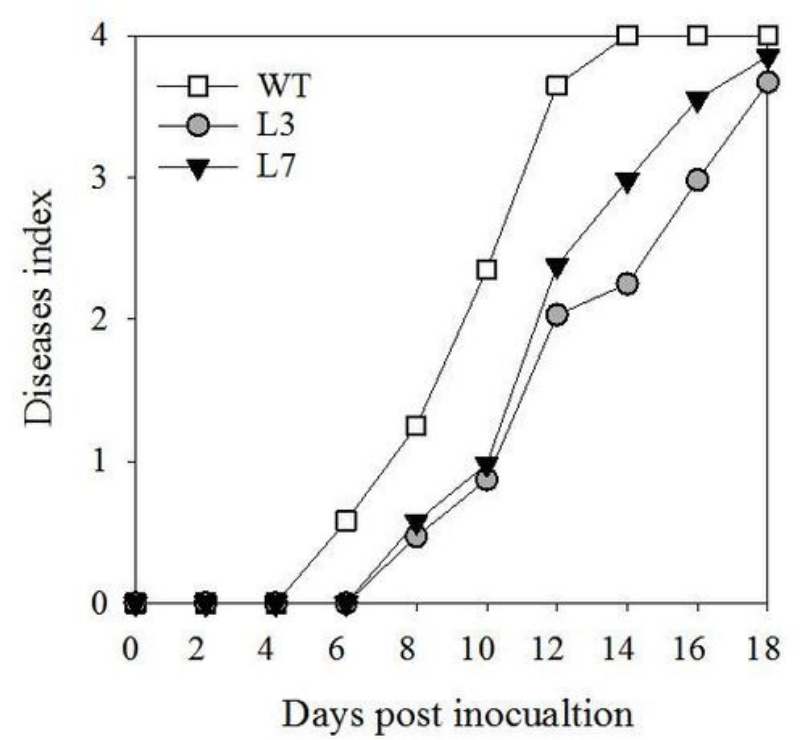

b

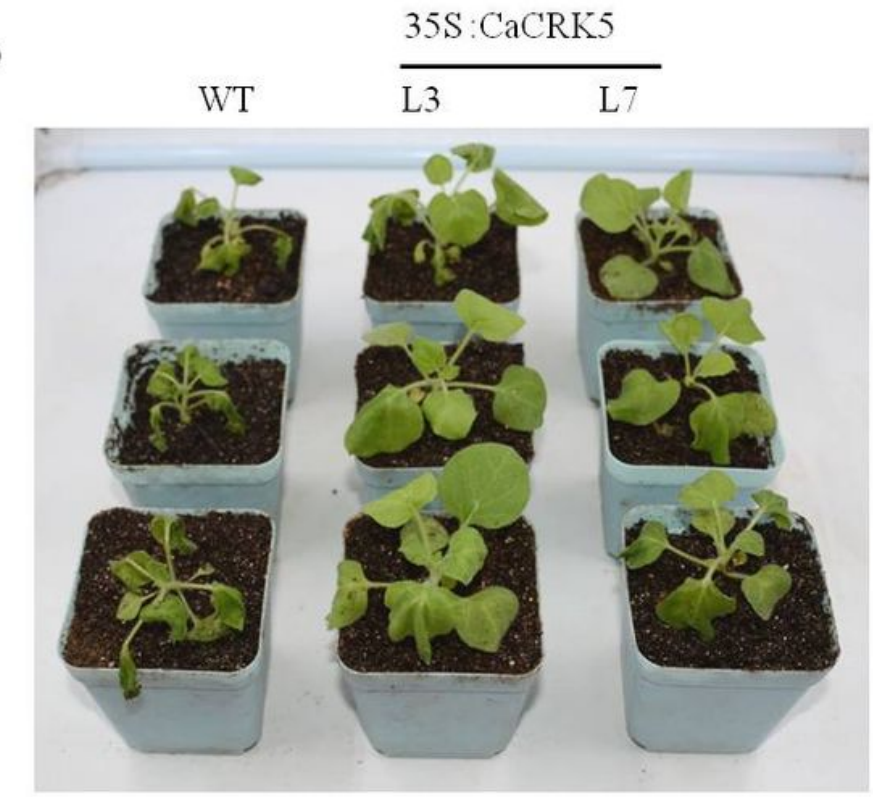

d

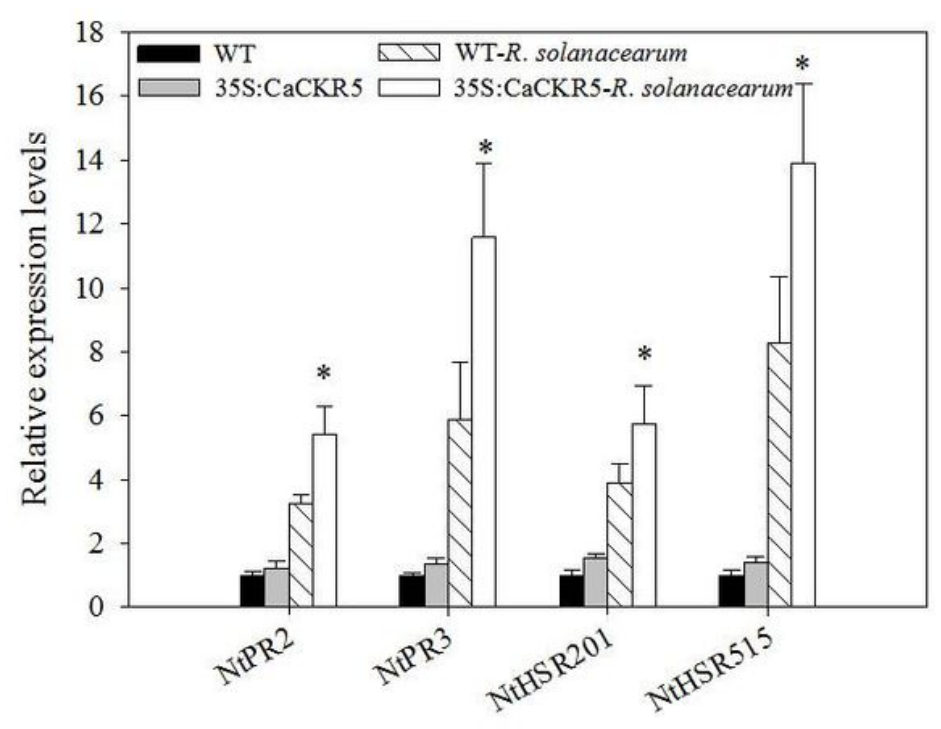

Figure 6 
Effect of CaCRK5 overexpression in tobacco on bacterial wilt disease by inoculation with R. solanacearum. a Expression levels of CaCRK5 in WT (wild-type) and transgenic tobacco lines (L3 and L7). b Symptoms in wild-type and overexpressed plants. Photograph was taken $12 \mathrm{~d}$ after rootinoculation with R. solanacearum. c Disease development of bacterial wilt was rated daily on a 0-4 disease index in wild-type and overexpressed plants. Each data point represents the mean disease index from three independent experiments containing 30 plants in total for each treatment. $d$ Expression of four defense related genes (NtPR2, NtPR3, NtHSR201 and NtHSR505) in the CaCRK5 overexpressing line (L3) and wild-type. Four-week-old wild-type and transgenic tobacco seedlings were leaf-inoculated with R. solanacearum. After $48 \mathrm{~h}$, total RNAs were extracted for qRT-PCR assays. Data represent means \pm SD ( $n=$ 3). Asterisks indicate significant differences according to Student's t test, ${ }^{*} P<0.05$.

a

Wildtype GTGCAGTCGAGCTAG CAATTATTG TAAACTTTATATTTA

Mutant GTGCAGTCGAGCTAG CAGGGGTTG TAAACTTTATATTTA b
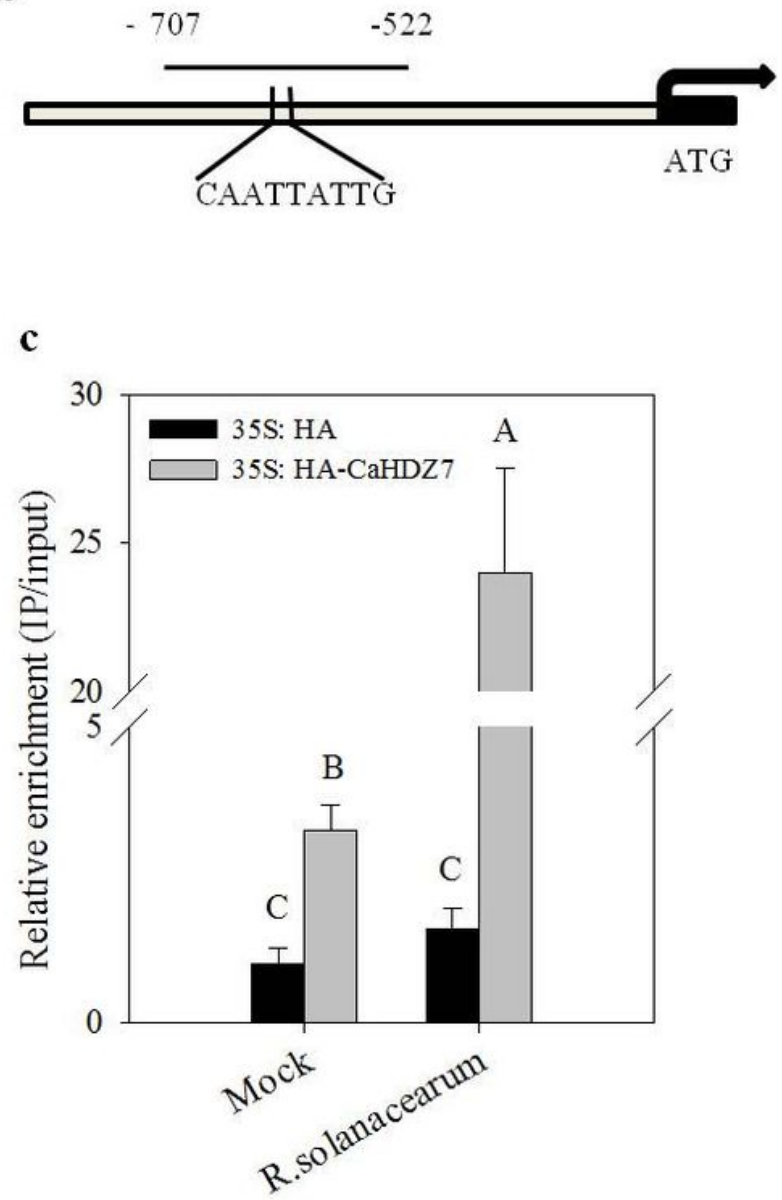

\section{Figure 7}

CaHDZ27 binds to the CAATTATTG motif in the promoter of CaCKR5. a EMSA was used to analyze the interaction of CaHDZ27-GST and a Cy5-labeled probe in vitro. The probe sequences containing the CAATTATTG motif in the CaCKR5 promoter are shown. CaHDZ27-GST purified protein was incubated with Cy5 fluorochrome labeled wild-type probe. Non labeled probes at 10- and 100-fold concentrations were added for the competition test. $b$ Schematic representation of the CaCKR5 promoter used for ChIPqPCR analysis. the blue lines indicate the sequence regions that were used for ChIP-qPCR. c, ChIP-qPCR 
assays showed the binding of CaHDZ27 to CaCKR5 promoter in vitro. DNA samples were coimmunoprecipitated with anti-HA antibody. The ChIP value was normalized to its respective input DNA value. Different letters indicate significant differences, as determined by LSD test $(P<0.01)$.
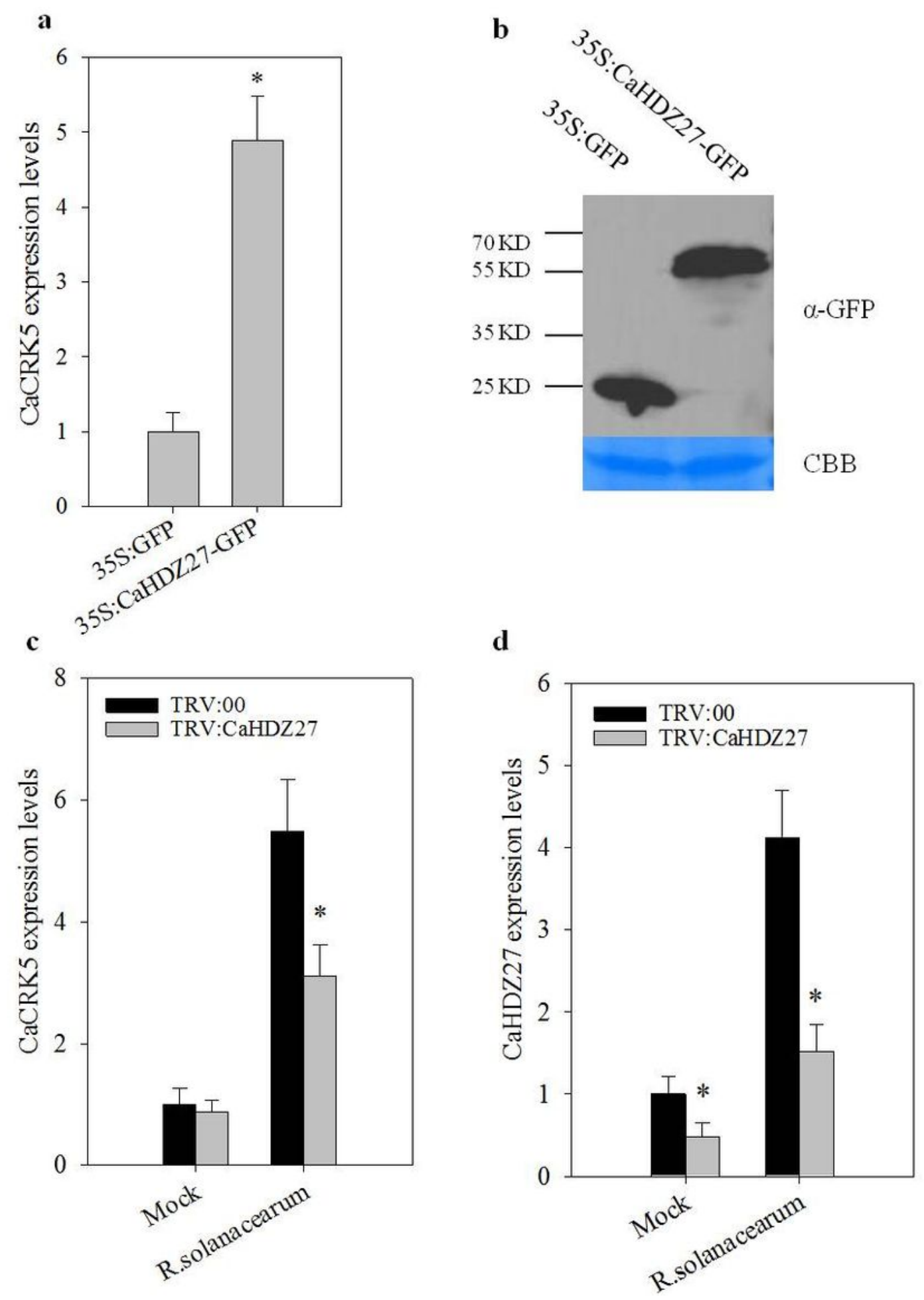

Figure 8

The effect of CaHDZ27 transit expression or scilence on expression of CaCKR5. a Relative transcript levels of CaCKR5 in transient expressed CaHDZ27 pepper leaves. b The success of CaHDZ27 transient 
expression in pepper leaves identified by western blotting $48 \mathrm{~h}$ post infiltration. c-d Relative transcript levels of CaCKR5 and CaHDZ27 in CaHDZ27 silenced plants by VIGS. Asterisks indicate significant differences at $P$ values $<0.05$.

a

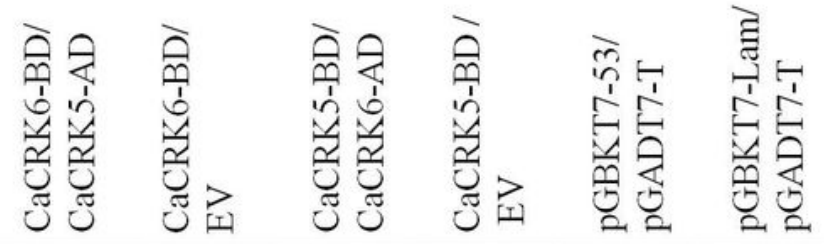

SD/-Trp/-Leu/-His/-Ade

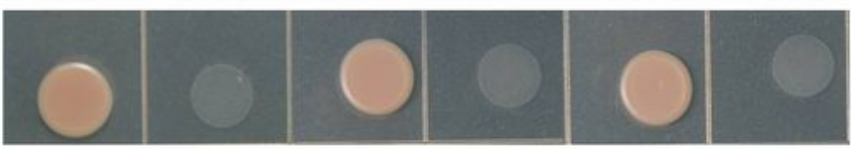

SD/-Trp/-Leu

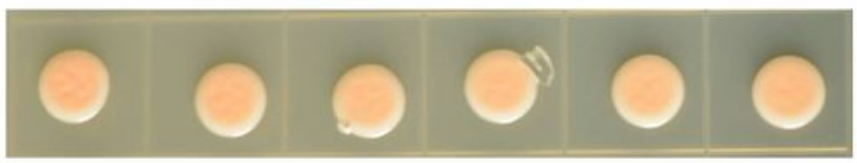

b
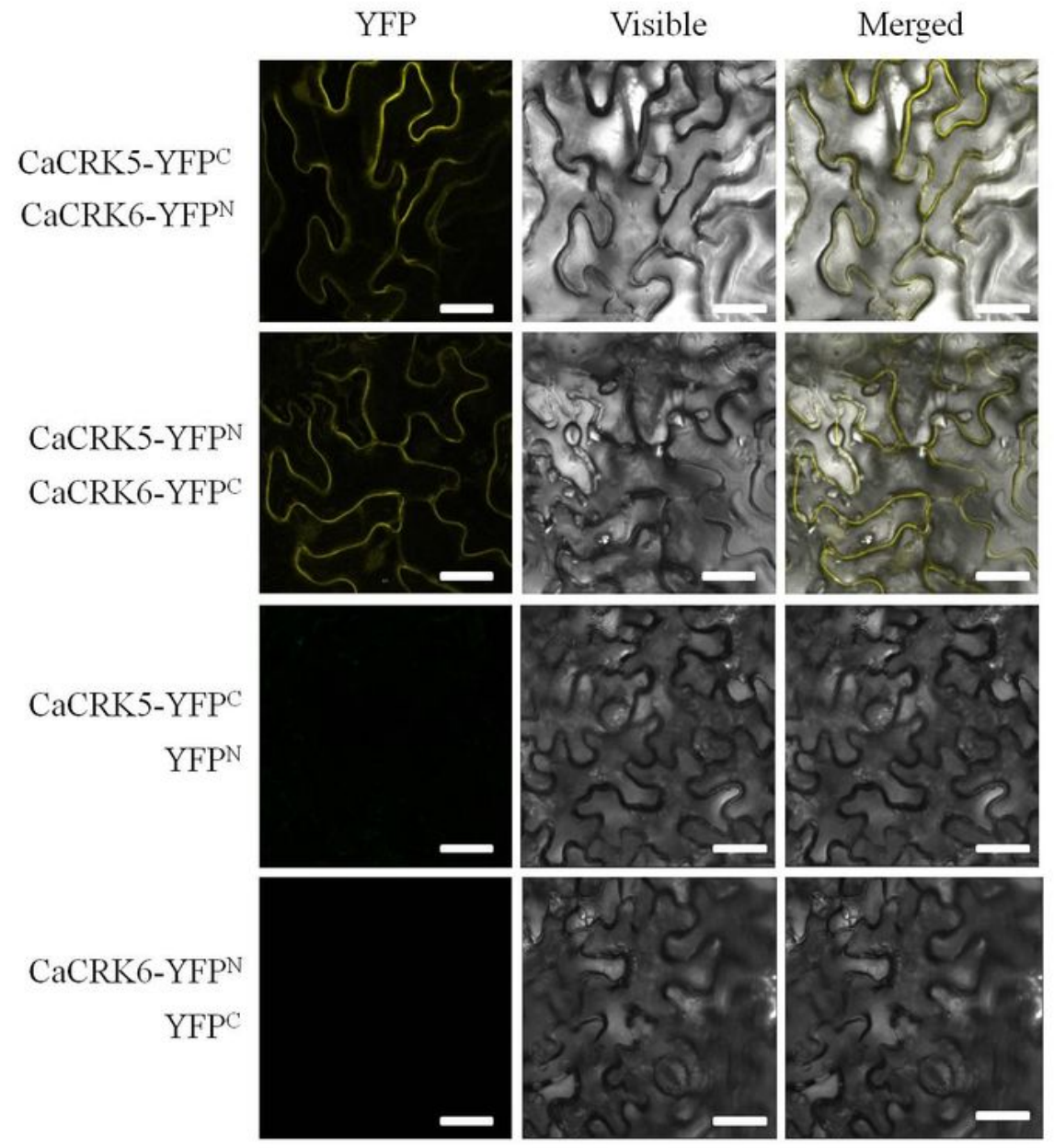

Merged

Figure 9

CaCRK5 interacts with CaCRK6. a Y2H assay. CaCRK5-BD and CaCRK6-AD were cotransformed into ' $\mathrm{Y} 2 \mathrm{H}$ Gold' yeast cells, which were grown on SD/-Trp-Leu and SD/-Trp-Leu-His-Ade for $3 \mathrm{~d}$. EV indicates the 
empty vectors for pGBKT7. b Bimolecular fluorescence complementation (BiFC) assays. YFP fusion constructs were transiently expressed in N.benthamiana leaves, and BiFC signals were observed under a fluorescence microscope. Bars $=50 \mu \mathrm{m}$. The experiment was repeated independently three times with similar result.

\section{Supplementary Files}

This is a list of supplementary files associated with this preprint. Click to download.

- Additionalfile1.docx

- Additionalfile2.jpg

- Additionalfile3.docx

- Additionalfile4.pdf 